\title{
Environmental electromagnetic characterization framework for wearable antenna materials
}

\author{
Frederick Declercq, Sam Agneessens, Hendrik Rogier \\ INTEC \\ Ghent University \\ Belgium, Ghent, 9000 \\ frederick.declercq@intec.ugent.be
}

\author{
Ivo Couckuyt, Tom Dhaene \\ iMinds \\ Ghent University \\ Belgium, Ghent, 9000 \\ ivo.couckuyt@ugent.be
}

\begin{abstract}
In wearable antenna design, the prevailing atmospheric conditions can have a significant effect on the electromagnetic properties of the fabric substrate and hence the resulting antenna performances. Therefore, an accurate characterization of this effect is an important issue in textile antenna design. This paper presents a dedicated constitutive parameter extraction method as a function of relative humidity of all materials used. The method relies on a comparison between measured and simulated antenna figure's of merit in order to extract complex permittivity of the substrate and effective bulk conductivity of the e-textile. A two-step approach is used for separating conductor losses from substrate losses. The problem of finding the best fit between simulated and measured data is solved by relying on a surrogate based optimization technique. Here, two fabric materials are characterized for relative humidity levels ranging from $10 \%$ to $90 \%$.
\end{abstract}

\section{INTRODUCTION}

Planar wearable antennas utilize textile fabric for the substrate and e-textile for the conductive planes [1]. As textile materials are sensitive to moisture, the surrounding environment will have an effect on their electromagnetic properties resulting in varying antenna performance. An accurate characterization of this effect aids the engineer in developing textile antennas capable of operating in all conditions. The effect of relative humidity (RH) on dielectric permittivity of textile materials exhibiting different sensitivities to moisture was investigated in [1] using a textile antenna resonance frequency perturbation technique. In [2], both a transmission line and cavity perturbation method were used to determine the complex permittivity of textile substrates as a function of RH and temperature.

In this paper, a dedicated characterization method for extracting complex permittivity of fabrics and conductivity of the e-textile as a function of $\mathrm{RH}$ is presented. The method is based on finding the best fit between simulated and measured antenna figures of merit. It relies on two distinct steps allowing us to separate substrate losses from conductor losses [3]. This inverse problem is converted into a forward optimization problem, which is solved by relying on a surrogate based optimization (SBO) technique.

\section{Characterization Process}

The goal of the characterization process is to extract permittivity $\epsilon_{r}$ and loss tangent $\tan \delta$ of the textile substrate and an effective bulk conductivity $\sigma$ of the e-textile. A flowchart of the characterization process, illustrating the two-step approach, is depicted in Fig.1.

First, only $\epsilon_{r}$ and $\tan \delta$ of the fabric are of interest. Starting from an estimated $\epsilon_{r}$, an inset-fed patch antenna using copper foil with known conductivity is designed to operate in the vicinity of $2.45 \mathrm{GHz}$. Agilent's Momentum is used for the computer-aided design of the antenna. For a fixed and known antenna geometry, bandwidth and resonance frequency $f_{r}$ derived from the frequency dependent reflection coefficient $\left|S_{11}\right|$, and antenna radiation efficiency $e_{r}$ depend on $\epsilon_{r}$ and $\tan \delta$ of the textile substrate. Hence, $\epsilon_{r}$ and $\tan \delta$ can be extracted by finding the best fit between simulated and measured antenna performances. To solve this problem, a cost function describing the discrepancy between measured and simulated data is defined as

$$
f=w_{1} \frac{1}{n} \sum_{i=1}^{n}\left(\left|S_{11}\right|_{i}^{d B}-\left.\widetilde{\mid S_{11}}\right|_{i} ^{d B}\right)^{2}+w_{2}\left|e_{r}\left(f_{r}\right)-\widetilde{e_{r}\left(f_{r}\right)}\right|,
$$

with $n$ the number of frequency points and $i$ the frequency index. Measurement data are represented by a tilde $(\cdot)$ whereas for simulation data no diacritical mark is used. To obtain a similar order of magnitude in both error terms of (1), the weighting factors are set to $w_{1}=1$ and $w_{2}=100$. [3] shows that using an error function that only compares $\left|S_{11}\right|$ data yields an inaccurate characterization of $\tan \delta$ in step 1 of the characterization process. Therefore, $S_{11}$ measurement data in the frequency range $2-3 \mathrm{GHz}$ are combined with the measured $e_{r}$ obtained with the generalized wheeler cap method [3]. This only requires one additional $S_{11}$ measurement with the antenna inside a properly designed copper cavity. Equation (1) is minimized by relying on an SBO technique in which the number of samples used for creating the surrogate model of (1) is limited to 70 [4]. The $\left(\epsilon_{r}, \tan \delta\right)$ corresponding with the minimum of (1) is the outcome of step 1.

Second, an e-textile based inset-fed patch antenna using the same textile substrate as in step 1 is designed and used for step 2 of the characterization process. During step 2, the $\tan \delta$ found in step 1 is used as a constant in the antenna simulation model. The properties of interest are $\epsilon_{r}$ of the substrate and $\sigma$ of the e-textile. Note that $\epsilon_{r}$ is still unknown since the use of e-textile results in an increased effective permittivity of the 
substrate compared to using homogeneous copper foil. This effect is accounted for by an equivalent $\epsilon_{r}$ in the simulation model. The $\left(\epsilon_{r}, \sigma\right)$ corresponding with the minimum of (1) is the outcome of step 2 .

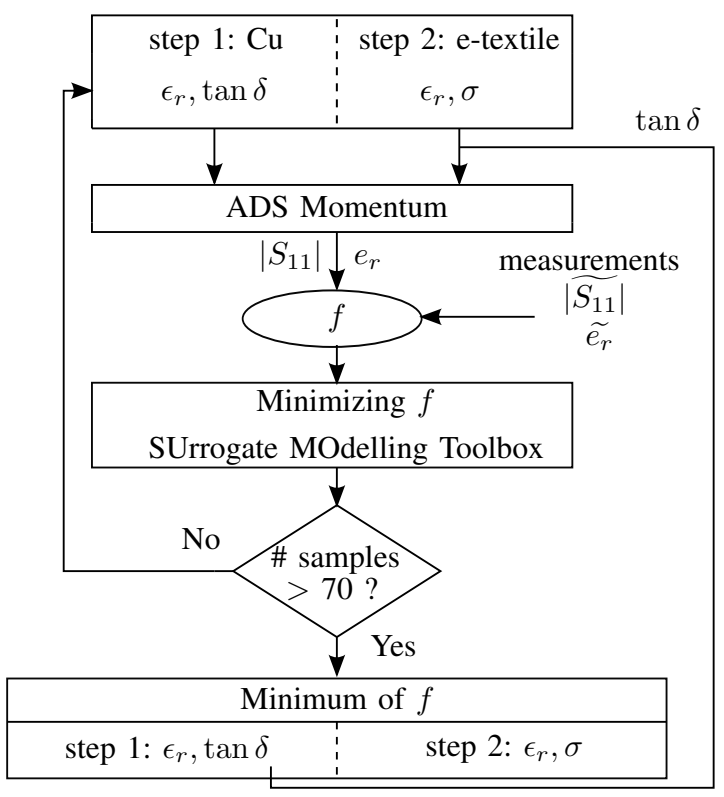

Fig. 1. Flowchart of the characterization process.

\section{Characterization Results}

\section{A. Materials, climatic chamber and optimization setup}

Two different materials, exhibiting different moisture regain $M R$ [3], and one e-textile were investigated. Fabric substrate 1 is a cotton/linen weave - based multilayered assembly with a thickness $h=2.4 \mathrm{~mm}$ and $M R=7.5$. Fabric substrate 2 is a polyaramide multilayered assembly with $h=1.67 \mathrm{~mm}$ and $M R=4.9$. The e-textile is a copper coated nylon fabric with a tarnish resistant finish for protection against oxidation. For each substrate, one copper based and one e-textile based antenna were designed.

All antenna prototypes were placed inside a climatic chamber and conditioned at one specific RH level for 24 hours at $23^{\circ} \mathrm{C}$. After 24 hours, an equilibrium state was reached between the moisture absorbed by the material and the moisture present in the climate chamber. Then the antenna prototypes were removed to perform the $S_{11}$ and $e_{r}\left(f_{r}\right)$ measurements according to the procedure described in Section II. This process was executed for RH-levels $10,20,30,40,50,60$, 70, 80 and $90 \%$.

For each RH-level, the $\left(\epsilon_{r}, \tan \delta\right)$ in step 1 and the $\left(\epsilon_{r}, \sigma\right)$ in step 2 were determined. The SBO algorithm is implemented in a flexible research platform called the SUrrogate Modelling (SUMO) Toolbox [4]. In step 1, the bounds are chosen $\epsilon_{r}=[1.5$ $2.5]$ and $\tan \delta=[0.0050 .1]$. In step 2 of the characterization process, the $\epsilon_{r}$-optimization bounds are identical as in step
1 and the optimization range for $\sigma=\left[5.0 \times 10^{3} \mathrm{~S} / \mathrm{m} 5.0 \times\right.$ $\left.10^{6} \mathrm{~S} / \mathrm{m}\right]$.

\section{B. Results}

For both fabrics, $\epsilon_{r}$ extracted in step 2 and $\tan \delta$ extracted in step 1 as a function of RH are given in Fig. 2. Both fabrics clearly exhibit different sensitivities to moisture absorption. Fabric 1 with the highest $M R$ undergoes the largest increase in $\epsilon_{r}$ and $\tan \delta$ for increasing $R H$. Since $\sigma$ is not affected by the moisture due to the tarnish resistant finish, an average $\sigma$ can be calculated over all measurements, equalling $7 \times 10^{5} \mathrm{~S} / \mathrm{m}$. The mean difference between measured $f_{r}$ and optimal simulated $f_{r}$ equals about $5 \mathrm{MHz}$ in step 1 and $4 \mathrm{MHz}$ in step 2. The mean $e_{r}$ difference between measured and optimal simulated $e_{r}$ equals about $1 \%$ and $5 \%$ in step 1 and step 2 , respectively.

\section{CONCLUSION}

A characterization framework was presented to determine the electromagnetic properties of wearable antenna materials as a function of relative humidity. The method requires only two reflection coefficient measurements yielding fast measurement times which makes the technique ideal for combination with climatic chambers. It was shown that the surrogate based optimization techniques yields an excellent agreement between measured and simulated data. The uncertainty in the extracted electromagnetic properties result from simplifications of the simulation model and measurement errors.

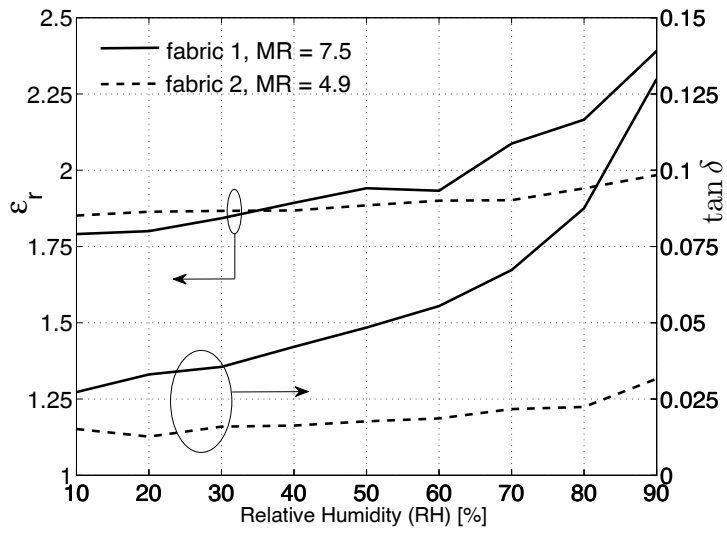

Fig. 2. Extracted $\epsilon_{r}$ and $\tan \delta$ of the substrates as a function of RH.

\section{REFERENCES}

[1] C. Hertleer, A. Van Laere, H. Rogier, and L. Van Langenhove, "Influence of relative humiditiy on textile antenna performance," Tex Res J, vol. 2, pp. 177-183, 2010.

[2] J. Lilja and P. Salonen, "Textile material characterization for softwear antennas," in Proceedings of the 28th IEEE conference on Military communications, Piscataway, NJ, USA, 2009, pp. 628-634.

[3] F. Declercq, I. Couckuyt, H. Rogier, and T. Dhaene, "Environmental high frequency characterization of fabrics based on a novel surrogate modelling antenna technique," Antennas and Propagation, IEEE Transactions on, vol. 61, no. 10, pp. 5200-5213, 2013.

[4] I. Couckuyt, F. Declercq, T. Dhaene, H. Rogier, and L. Knockaert, "Surrogate-based infill optimization applied to electromagnetic problems," Int. J. RF Microw. Comput.-Aided Eng., vol. 20, no. 5, pp. 492-501, Sept. 2010 